\title{
Mortality, Fertility, and the OY Ratio in a Model Hunter-Gatherer System
}

\author{
Andrew A. White* \\ University of Michigan, Ann Arbor, MI \\ KEY WORDS paleodemography; demography; longevity; agent-based model; complex \\ systems theory
}

\begin{abstract}
An agent-based model (ABM) is used to explore how the ratio of old to young adults (the OY ratio) in a sample of dead individuals is related to aspects of mortality, fertility, and longevity experienced by the living population from which the sample was drawn. The ABM features representations of rules, behaviors, and constraints that affect personand household-level decisions about marriage, reproduction, and infant mortality in hunter-gatherer systems. The demographic characteristics of the larger model system emerge through human-level interactions playing out in the context of "global" parameters that can be adjusted to produce a range of mortality and fertility conditions. Model data show a relationship between the OY ratios of living populations (the living OY ratio) and assemblages of dead individuals
\end{abstract}

The OY ratio is the ratio of older to younger adults in a population or sample, developed by Caspari and Lee (2004) as a simple metric to evaluate ideas about the timing of gross changes in patterns of life history and adult longevity over the course of human evolution. Calculation of this measure involves assigning individuals to "young adult" and "old adult" categories based on patterns of molar eruption and wear. The occlusal eruption of M3 is taken as the end of the juvenile period and the beginning of adulthood (i.e., about age 15 years). Patterns of relative molar wear were used to categorize individual fossils with erupted third molars as either "young adult" (between the ages of 15 and 30 years) or "old adult" (over the age of 30 years). Because the "old" adult category includes individuals twice as old as the "young" adult category, "old" adults have the potential to be grandparents.

Caspari and Lee (2004:10898) interpreted differences in the OY ratios of samples of australopithecines, early Homo, Neanderthals, and early Upper Paleolithic humans as reflecting a temporal trend toward increasing survivorship of older adults during the course of human evolution, with the most dramatic increase in longevity in the Upper Paleolithic. This conclusion was the subject of some debate (see Caspari and Lee, 2005a, 2005b; Hawkes and O'Connell, 2005; Minichillo, 2005), as it conflicted with the idea that longer adult lifespans were important to the evolution and success of Homo erectus (e.g., O'Connell et al., 1999). This disagreement highlighted a need to clarify the relationship between the OY ratio of a skeletal/fossil assemblage and the demographic characteristics of the living population from which that assemblage was drawn. drawn from those populations (the dead OY ratio) that is consistent with that from empirically known ethnographic hunter-gatherer cases. The dead OY ratio is clearly related to the mean ages, mean adult mortality rates, and mean total fertility rates experienced by living populations in the model. Sample size exerts a strong effect on the accuracy with which the calculated dead OY ratio reflects the actual dead OY ratio of the complete assemblage. These results demonstrate that the dead OY ratio is a potentially useful metric for paleodemographic analysis of changes in mortality and mean age, and suggest that, in general, huntergatherer populations with higher mortality, higher fertility, and lower mean ages are characterized by lower dead OY ratios. Am J Phys Anthropol 154:222-231, 2014. () 2014 Wiley Periodicals, Inc.

I use an agent-based model (ABM) to explore this issue. Agent-based modeling is a form of modeling in which the behavior observable at the system level is "generated from the bottom of the system by the direct interactions of the entities that form the basis of the model" (Miller and Page, 2007:66). An ABM is a useful tool for linking the small scales of human behavior that we can observe ethnographically to the population-level characteristics we seek to understand based on assemblages of fossils, skeletons, or other material remains. Through systematic experimentation with model systems, cause-effect relationships among human-level behaviors and the systemlevel characteristics that emerge as a result of those behaviors can be investigated as empirical problems rather than simply assumed or estimated through comparison of different ethnographic cases.

An ABM representing a hunter-gatherer system is used here to understand how the demographic

Additional Supporting Information may be found in the online version of this article.

*Correspondence to: Andrew A. White, Department of Anthropology, 101 West Hall, 1085 S. University Ave., Ann Arbor, MI 48109. E-mail: aawhite@umich.edu.

Received 7 September 2013; revised 13 January 2014; accepted 3 February 2014

DOI: 10.1002/ajpa.22495

Published online 13 February 2014 in Wiley Online Library (wileyonlinelibrary.com). 
characteristics of "living" populations, such as mortality, fertility, and mean age, are related to both the OY ratio of the population when it is alive (i.e., the living OY ratio) and the OY ratio of assemblages of dead persons produced by the population (i.e., the dead OY ratio). Experiments show that the dead OY ratio is clearly related to the mortality and fertility experienced by living populations as well as the mean age of the population. Although my analysis does not directly address the conclusions of Caspari and Lee (2004) with respect to the evolutionary timing of increases in human longevity, my results are consistent with the idea that lower dead OY ratios are generally associated with living populations with lower mean ages (i.e., containing fewer older adults).

\section{AGENT-BASED MODELING AND COMPLEX SYSTEMS THEORY AS TOOLS FOR PALEODEMOGRAPHY}

The demographic characteristics of a living population are the result of numerous human-level interactions and behaviors: persons and households make decisions about marriage and reproduction based on their individual circumstances within the context of "global" conditions that exert effects and constraints on all members of the population (e.g., the physiological factors that govern the length of the female reproductive span, ecological circumstances that affect the contributions of children to subsistence, cultural rules affecting marriage behaviors, etc.). Some demographic characteristics of living populations, such as age distribution, can be measured at a single point in time. Others, such as rates of mortality or fertility, are dependent on information collected over a span of time.

The characteristics of an assemblage of dead individuals (e.g., the fossils or skeletons of all or some of the members of a population) are the result of the same processes of mortality and fertility that operated in the living population. Unlike in a living population, however, the age distribution of a dead assemblage is a composite of mortality events that occurred over some length of time. The age distribution of a dead assemblage of individuals does not necessarily directly correspond to the age distribution of the living population from which it was drawn (see Angel, 1969; Horowitz et al., 1988; Paine, 1989; Konigsberg and Frankenberg, 1994; Caspari and Lee, 2005b). Understanding the demographic implications of the age distributions of assemblages of dead individuals remains a fundamental challenge of paleodemography (e.g., Roksandic and Armstrong, 2011).

Complex systems theory and agent-based modeling offer an avenue for addressing this challenge. The term "complex system" here refers to any system with the particular suite of characteristics where system-level behavior can be understood as emerging "bottom up" from the interactions and behaviors of individual agents. A complex system, in general, has three basic characteristics: (1) it consists of a relatively large number of interacting agents; (2) it exhibits emergent behavior (self-organizing, collective behavior difficult to anticipate from knowledge of the individual agents' behavior); and (3) this emergent behavior does not result from central control (Boccara, 2004:3). Hunter-gatherer societies are complex systems in that they are composed of numerous individuals who act, react, and interact on local scales. The demographic characteristics of these systems (e.g., population age structure, mean fertility, mean mortality) emerge through a large number of human-level interactions and behaviors related to marriage, reproduction, and mortality.

ABMs are suitable tools for understanding complex systems because they allow one to represent the humanlevel behaviors, interactions, and constraints that are important to the system at the levels where they actually operate. Because agents (representing individual people in the modeling efforts discussed here) are discrete entities, a heterogeneous population of agents can interact according to a set of parameters and rules. Experiments can be conducted to investigate causeeffect relationships between behaviors at different levels of a system. Model data, derived from a system with known rules, parameters, and behavior, can be compared to observations made in the real world. This provides us with an avenue toward the explanation of real-world phenomena that hinges on the ability of an ABM to generate, under known conditions, some set of behaviors we are interested in understanding. This is what Epstein (2006) terms "generative social science."

Use of an ABM as a tool for paleodemography has several advantages over the equation-based models that are often employed to interpret fossil or skeletal assemblages. In contrast to equation-based models, $\mathrm{ABM}$ s allow the representation of heterogeneity within a population, the operation of stochastic processes at the appropriate level in a system, and the potential of exploring linkages between lower-level behaviors and the characteristics of the system that emerge from those behaviors. It also allows us to dispense with some of the principal assumptions that are required for equation-based models to be applied. The assumptions of infinite populations, stable population size, fixed/homogenous birth/death rates, and/or random mating that are often (by necessity) incorporated into equationbased models limit the utility of those models for exploring demography in populations where human-level interactions and stochastic processes are an important factor (cf. Wobst, 1975:76). The conditions of stable population theory are probably violated by almost all human populations that are the object of demographic or paleodemographic study (cf. Hill and Hurtado, 1996:116).

$\mathrm{ABM}_{\mathrm{s}}$ allow the relationships between the characteristics of a model population, the constituent components of that population, and the factors affecting the population to be examined at many levels of detail. This allows one to do two important things: (1) understand how the model system itself behaves (allowing the model to be "validated" by comparison to the behavior of real systems) and (2) derive outputs that are suitable for comparison to the physical remains that constitute the direct evidence of past human biological and/or cultural behavior and understand the relationship between those outputs and the characteristics and behaviors of the model system that produced them. An experimental framework can be employed where the behavioral rules or parameters affecting the model system can be changed and the effects of those changes on the characteristics of the system and the calculated outputs (such as the OY ratio) observed. This allows us to use an ABM as a tool for building explanatory theory linking patterns that we may observe in the physical world to causes that are unobservable but understandable.

\section{THE MODEL}

The model used for this analysis is Version 2 of the ForagerNet3_Demography model (hereafter FN3D_V2). 
This is a nonspatial ABM designed to serve as a platform for exploring hunter-gatherer demography. It is a development from the ForagerNet2 (White, 2012) and FamilyNet2 (White, 2013) models. It is a generalized model that is not intended to exhaustively represent all details of any given hunter-gatherer system. The exclusion of extraneous detail is a purposeful strategy to aid in constructing a model whose structure and behavior are understandable and potentially relevant to many cases. In the terminology of Gilbert (2008), FN3D_V2 is a "middle range" model that aims "to describe the characteristics of a particular social phenomenon, but in a sufficiently general way that their conclusions can be applied" to many examples of the same phenomenon (Gilbert, 2008:42).

The FN3D_V2 model was written in Java programming language and built using Repast J. Repast (Recursive Porous Agent Simulation Toolkit) is a free, opensource agent-based modeling and simulation toolkit that was created at the University of Chicago in collaboration with Argonne National Laboratory (North et al., 2006). The raw code for the FN3D_V2 model and detailed descriptions of its classes, variables, parameters, structure, and operation are provided as supporting information to this article and elsewhere online (www.openabm. org/model/4087/version/1/view). Documentation of Repast can be found at www.repast.sourceforge.net. This section provides a necessarily brief overview of the design and operation of the model augmented by a short description of factors affecting fertility, mortality, and stable population size in the model.

\section{General design and operation}

The FN3D_V2 model has three main "levels": person, household, and system. Each agent in the model represents an individual person who is a discrete entity with a unique identity. Households are co-residential groupings of persons that form through marriage and change in size and composition primarily through marriage, reproduction, and mortality. Social links define relationships between pairs of living persons and are used to enforce marriage prohibitions. The system of the model is composed of all persons and households in existence at a given point in time. Model-level parameters set conditions for all persons or all households in the world and define aspects of the system: for example, all persons become eligible to marry at the same age. There is no spatial component to interaction and behavior, eliminating the potential effects of information flow, mobility, and population density on the analysis performed here.

Methods are named sections or "chunks" of code that perform a sequence of operations when called. Methods representing marriage, reproduction, and death operate at the person and household levels in this model. Individual persons and households make probabilistic deci- sions about reproduction, marriage, and infanticide based on the current dependency ratio of the household (the ratio of the number of consumers to the number of producers in the household). Although the base probabilities affecting reproduction and mortality are set by model-level parameters (i.e., they are the same across the population), the economic circumstances of individual households affect the behavior of individuals in those households on a case-by-case, step-by-step basis (see below).

At the start of each run, the model produces an initial population of a specified number of persons of random sex and random age between 15 (the age at which the potential for reproduction begins) and 20 years. The initial households in a model run are created through marriages between eligible males and females in this initial population. A model run starts with an initial population of reproductive-age adults rather than a "realistic" population-age distribution in order to allow the characteristics of the living population to emerge through personand household-level interactions and behaviors.

Following the creation of the initial population, time passes in the form of discrete steps. Each step represents 1 week (5200 steps representing 100 years). At each step, the model initiates a sequence of operations that includes the methods for marriage, reproduction, and death. This same sequence of operations is repeated in every subsequent step until the model has completed a specified number of steps. Each model run is 1000 years $(52,000$ steps $)$ in duration. The first 500 years of each run are a stabilization period during which the size and structure of the population emerge. Data required for calculating relevant demographic variables are collected during the second 500 years of the run. Summary data are produced at the end of a run and appended to a data file for analysis.

The validity of a model (how well the model represents what it is intended to represent) can be evaluated by comparing the behaviors of the model with the known behaviors of the real-world systems it purports to represent (see Gilbert, 2008). A summary of ethnographic data on hunter-gatherer fertility, infant mortality, marriage age, and mean family size is presented in Table 1. Summary data from the experiments discussed below ( $n=2000$ runs) demonstrate that, at the parameter settings utilized for this article, the model produces distributions of values for these variables that overlap the ethnographic ranges (Fig. 1). These are system-level characteristics that are the result of the interplay between person- and household-level interactions and behaviors and the model-level "rules" and constraints that influence marriage, reproduction, and mortality. The households that form within the model systems are verifiably consistent with those documented among ethnographic hunter-gatherers in terms of their size, composition, and developmental cycles (see White, 2013:157-158).

TABLE 1. Summary of ethnographic data on hunter-gatherer fertility, mortality, and marriage age

\begin{tabular}{lccl}
\hline Variable & Range & Approximate mean & \multicolumn{1}{c}{ Reference(s) } \\
\hline Total fertility rate & $2.6-8.0$ births & 5.4 births & Hewlett 1991: Table 2; Pennington 2001: Table 7.2 \\
Inter-birth interval & $2.5-4.0$ years & - & Kelly 1995: Table 6.7; Pennington 2001: Table 7.4 \\
Intensity of polygyny & $0-10$ wives & - & Betzig 1986; Keen 2006 \\
Infant mortality & $10-30$ percent & 20 percent & Hewlett 1991: Table 3; Kelly 1995: Table 6.9 \\
Female age at marriage & $5-22$ years & 14 years & Binford 2001: Table 8.07 \\
Male age at marriage & $12-35$ years & 21 years & Binford 2001: Table 8.07 \\
\hline
\end{tabular}




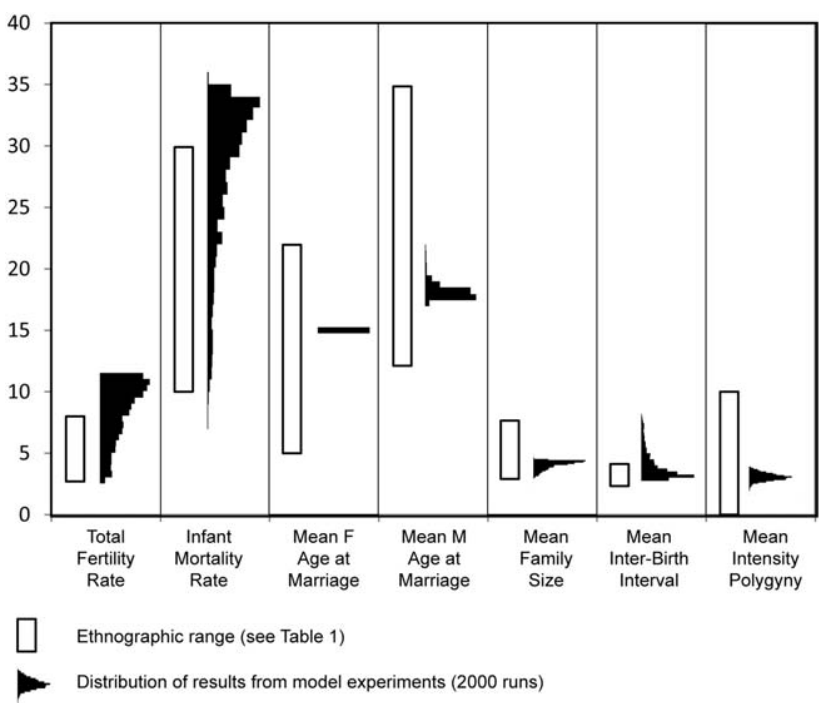

Fig. 1. Comparison between ranges of demographic variables in ethnographic cases and in the model dataset.

These points of consistency suggest that the FN3D_V2 model reasonably captures many of the relevant aspects of hunter-gatherer systems that are of interest here, and is therefore a useful tool for investigating relationships between the OY ratio and the demographic characteristics of living populations.

\section{Fertility, mortality, and stability of population size}

Fertility, mortality, and population size in the FN3D_V2 model are affected by: (1) the values of modellevel parameters that apply to the entire population; (2) the person- and household-level behaviors and interactions; and (3) the feedbacks among various components of the model. This section describes how fertility and mortality are represented in the model and how population size is regulated.

Fertility and reproduction. Following the start of a model run, new persons in the population are created through reproduction. Only married females who are neither currently pregnant nor in a period of postpartum amenorrhea are eligible to become pregnant. The maximum period of post-partum amenorrhea (a period of infertility following childbirth) is set by the value of the parameter $\max P P A$. When $\max P P A=72$ weeks (18 months), the probability that a female will return to a fertile state each week following childbirth is calculated as 1/72 (0.0139). Females who remain infertile during this period automatically regain fertility after 72 weeks.

A female's potential fertile period occurs between the ages of 11 and 55 years. The yearly base probabilities of a married, fertile female becoming pregnant are agespecific and follow a pattern similar to that documented for the !Kung (Howell, 1979) and the Ache (Hill and Hurtado, 1996) (Table 2). These base probabilities are adjusted by multiplication with the value of a modellevel parameter (fertilityMultiplier). For a 24-year-old female, for example, the base probability of pregnancy each step of her 24th year is 0.00673 if the value of fertilityMultiplier is set to $1.4(0.25 \times 1.4 / 52)$. The fertility-
TABLE 2. Age-specific yearly base probabilities of pregnancy and death

\begin{tabular}{lcc}
\hline $\begin{array}{l}\text { Age category } \\
\text { (years) }\end{array}$ & $\begin{array}{c}\text { Base probability of } \\
\text { pregnancy }\end{array}$ & $\begin{array}{c}\text { Base probability of } \\
\text { death }\end{array}$ \\
\hline 0 & 0 & 0.07 \\
1 & 0 & 0.07 \\
2 & 0 & 0.06 \\
3 & 0 & 0.05 \\
4 & 0 & 0.04 \\
5 & 0 & 0.03 \\
$6-10$ & 0 & 0.02 \\
$11-15$ & 0.01 & 0.015 \\
$16-20$ & 0.15 & 0.015 \\
$21-25$ & 0.25 & 0.015 \\
$26-30$ & 0.28 & 0.015 \\
$31-35$ & 0.28 & 0.015 \\
$36-40$ & 0.25 & 0.015 \\
$41-45$ & 0.15 & 0.018 \\
$46-50$ & 0.08 & 0.02 \\
$51-55$ & 0.01 & 0.03 \\
$56-60$ & 0 & 0.04 \\
$61-65$ & 0 & 0.08 \\
$66-70$ & 0 & 0.12 \\
$71-75$ & 0 & 0.20 \\
$76-80$ & 0 & 0.30 \\
$81-85$ & 0 & 0.30 \\
$>85$ & 0 & 1.00 \\
\hline
\end{tabular}

Multiplier parameter can be used to adjust the model through a continuous range of low- to high-fertility conditions while maintaining the shape of the age-specific curve.

The probability of pregnancy is reduced if the addition of another child would raise the household's dependency ratio above 1.75 . This represents the existence of mechanisms for avoiding pregnancy based on household economics. The chance of avoiding pregnancy is determined by calculating how much above 1.75 the dependency ratio would rise if another child were to be added and taking this amount as a percentage of 1.75 (e.g., the chance of avoidance is 100 percent if another child would raise the dependency ratio of the household to 3.5). Successful reproduction results in the creation of a child of random sex who is then added to the household.

The reproduction methods also include a mechanism for terminating the life of a newborn infant (i.e., committing infanticide). The chance of infanticide is calculated using the dependency ratio in the same way as avoidance of procreation: the difference is that the birth and subsequent death of a child figure into infant mortality rates where avoidance of procreation does not. The sex of a child does not affect the probability of infanticide in the model.

Mortality. Each person is exposed to a risk of death at each step. The yearly base probabilities of death are agespecific and follow a pattern similar to that documented for the Ache (Hill and Hurtado, 1996) and the Tsimane (Gurven and Kaplan, 2007) (see Table 2). If a person reaches a certain maximum age (set by the value of the parameter maxAge, 86 for the experiments discussed here), death is automatic. The base yearly probabilities of death are adjusted by multiplication with the value of a model-level parameter (mortalityMultiplier) that can be used to produce a continuous range of low- to highmortality conditions while maintaining the shape of the age-specific curve. Infants can experience increased 
mortality rates through the economically sensitive infanticide mechanism that is represented in the model (see above).

Stability of population size. The model uses a feedback between population size and death probabilities to stabilize population size. A model-level parameter ( $p o p$ MortAdjustPoint) specifies the population size above which probabilities of death are increased and below which probabilities of death are decreased. Each step, the model adjusts death probabilities by comparing the current size of the population to the size of the population specified by popMortAdjustPoint. If the current population is 605, for example, and popMortAdjustPoint = 500 , the probability of death for any given person that step is multiplied by 1.21 (605/500).

Note that a popMortAdjustPoint of 500 does not mean that the population will stabilize in size around 500: it simply means that the base probabilities of death are positively or negatively adjusted based on whether population size is above or below 500. At a given value of popMortAdjustPoint, the particular size at which a model population stabilizes is a product of the balance between fertility and mortality. Populations with relatively low rates of fertility and high mortality tend to stabilize at sizes significantly below popMortAdjustPoint, whereas populations with high-fertility rates and low mortality tend to stabilize at sizes well above popMortAdjustPoint.

\section{EXPERIMENTS}

A series of experiments was used to explore the relationships between mortality, fertility, and OY ratios of

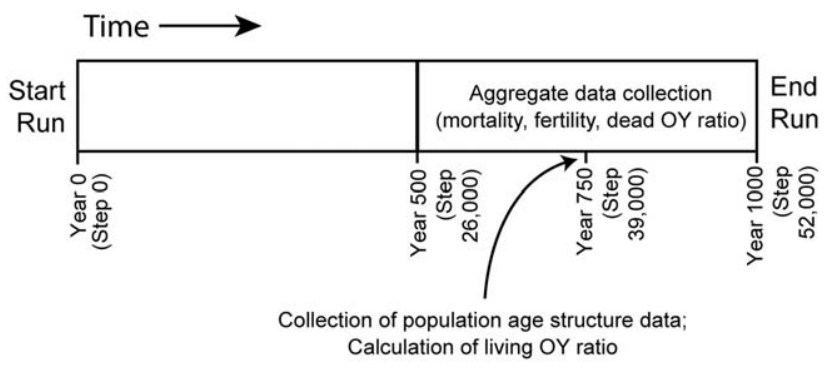

Fig. 2. Structure of model experiments. living populations and assemblages of dead individuals produced by those populations. Experiments included a total of 2000 runs, each 1000 years in duration (Fig. 2). Populations in eight runs went extinct and are excluded from analysis. The values of key model-level parameters are listed in Table 3.

The model was configured to set the values of the fertilityMultiplier and mortalityMultiplier parameters to independent, random numbers between 0.5 and 6 in order to generate a range of combinations of fertility and mortality conditions. The actual mortality and fertility rates experienced by a population were the result of model systems finding a balance between mortality and fertility that allowed a relatively stable population size with plausible household-level characteristics. The actual infant (age 0 years), child (age $2-10$ years), and adult (age 16-50 years) mortality rates experienced by the population during the 500-year data collection period were recorded. The mean total fertility rate during the data collection period was calculated as the mean number of live births per female who survived the majority of her reproductive years (defined as survival to age 45 years).

The OY ratio was defined, following Caspari and Lee (2004), as the ratio of "old" adults (over twice minimum reproductive age) to "young" adults (between minimum reproductive age and twice minimum reproductive age). With the ageAtMaturity set to 15 , this translates into groups of "old adults" over 30 years of age and "young adults" between the ages of 15 and 30 years.

The living OY ratio was calculated halfway through the 500-year data collection period by counting the numbers of old and young adults in the living population at step 39,000. The mean age of the living population was calculated simply by averaging the ages of all living members of the population.

The dead OY ratio was calculated by preserving a list of all the young and old adults who died during the 500year data collection period. Age categories used for the dead OY ratio were the same as those used to calculate the living OY ratio. At the end of a run, the dead OY ratio was calculated based on the complete dead assemblage of individuals as well as a series of samples of varying size $(10,50,100,250,500,750$, and 1000 individuals) drawn from that complete assemblage. These samples were produced by randomly selecting the required number of individuals from the list of all dead persons from a given run.

TABLE 3. Values of key parameters in experiments

\begin{tabular}{|c|c|c|}
\hline Parameter & Description & Value \\
\hline ageAtMaturity & $\begin{array}{l}\text { Age (in years) at which a person is eligible to marry (and therefore eligible } \\
\text { to reproduce) }\end{array}$ & 15 (constant) \\
\hline agetAtProduction & $\begin{array}{l}\text { Age (in years) at which a person is counted as a "producer" for purposes of } \\
\text { calculating the dependency ratio of a household }\end{array}$ & 14 (constant) \\
\hline $\operatorname{maxAge}$ & Maximum age (in years) a person may attain & 86 (constant) \\
\hline popMortAdjustPoint & $\begin{array}{l}\text { Population size above which base probabilities of mortality are increased } \\
\text { and below which base probabilities of mortality are decreased }\end{array}$ & 500 (constant) \\
\hline $\max P P A$ & Maximum duration (in weeks) of post-partum amenorrhea & 72 (constant) \\
\hline sustainableCP & $\begin{array}{l}\text { Value of dependency ratio considered "normal"; a dependency ratio }> \\
\text { sustainableCP has positive effect on probabilities of avoiding reproduction } \\
\text { or committing infanticide }\end{array}$ & $1.75^{\mathrm{a}}$ (constant) \\
\hline fertilityMultiplier & Adjusts the base age-specific probabilities of pregnancy by a set factor & $0.5-6$ (random) \\
\hline mortalityMultiplier & Adjusts the base age-specific probabilities of death by a set factor & $0.5-6$ (random) \\
\hline
\end{tabular}

a A dependency ratio of 1.75 was chosen to represent "typical" hunter-gatherer households based on ethnographic data (Binford 2001:230). 


\section{RESULTS}

The results of the experiments are presented to address four interrelated issues: (1) the relationship between the living and dead OY ratios; (2) the relationships between mortality, fertility, and the living and

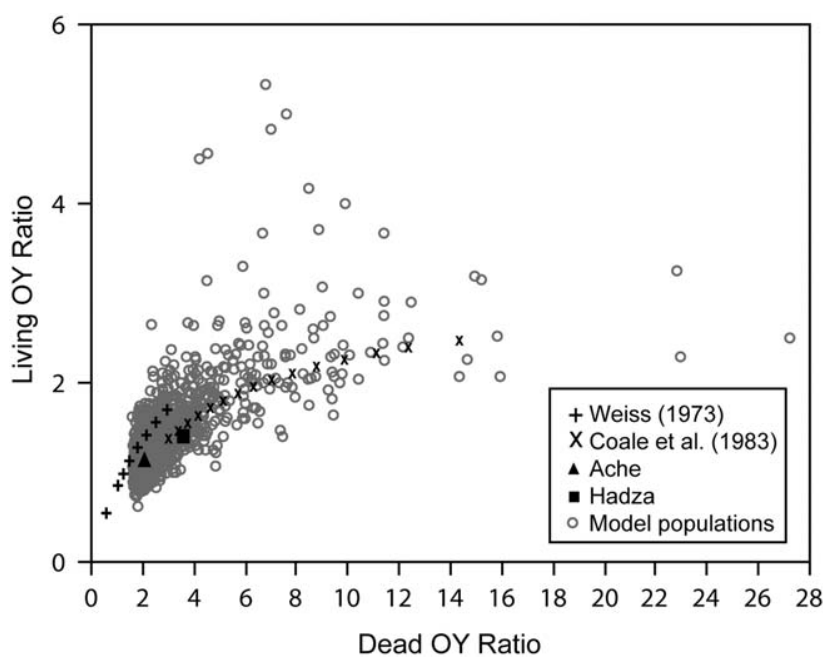

Fig. 3. Relationship between the living and dead OY ratios of model populations with data from the ethnographic populations shown by Caspari and Lee (2005b: Fig. 1) superimposed.
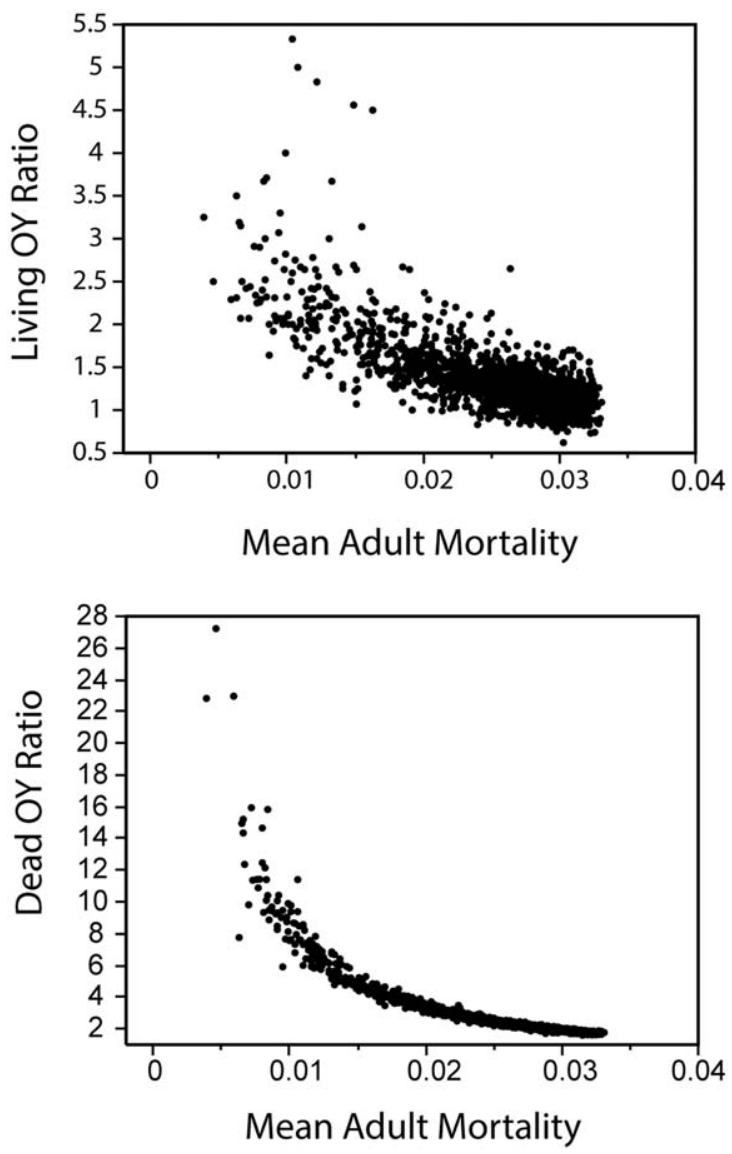

dead OY ratios; (3) the relationship between the mean age of the population and the living and dead OY ratios; and (4) the effects of sample size on the accuracy of the dead OY ratio. All data analyses were performed using JMP 11.0.0. The dataset used for the analysis is provided as supporting material.

\section{Relationship between the living and dead OY ratios}

The relationship between the living and dead OY ratios of model populations is shown in Figure 3. Data shown by Caspari and Lee (2005b:Fig. 1) are superimposed for comparison. The overall trend of the model data is very similar to that derived from the Weiss (1973) and Coale et al. (1983) data, and the cloud of model data overlaps with the single data points from the Hadza and the Ache. This suggests that the relationships between the living and dead OY ratios of model systems are like those from ethnographic cases in which values for both these measures can be empirically estimated. The spread of data points from the model shows that, while the living and dead OY ratios are related, the dead OY ratio is not a straightforward reflection of the OY ratio of the living population. Data produced by the model have a significantly larger range of values than ethnographic data. This is expected because the model runs include a broader range of mortality/fertility conditions than is represented among the small number of ethnographic cases.
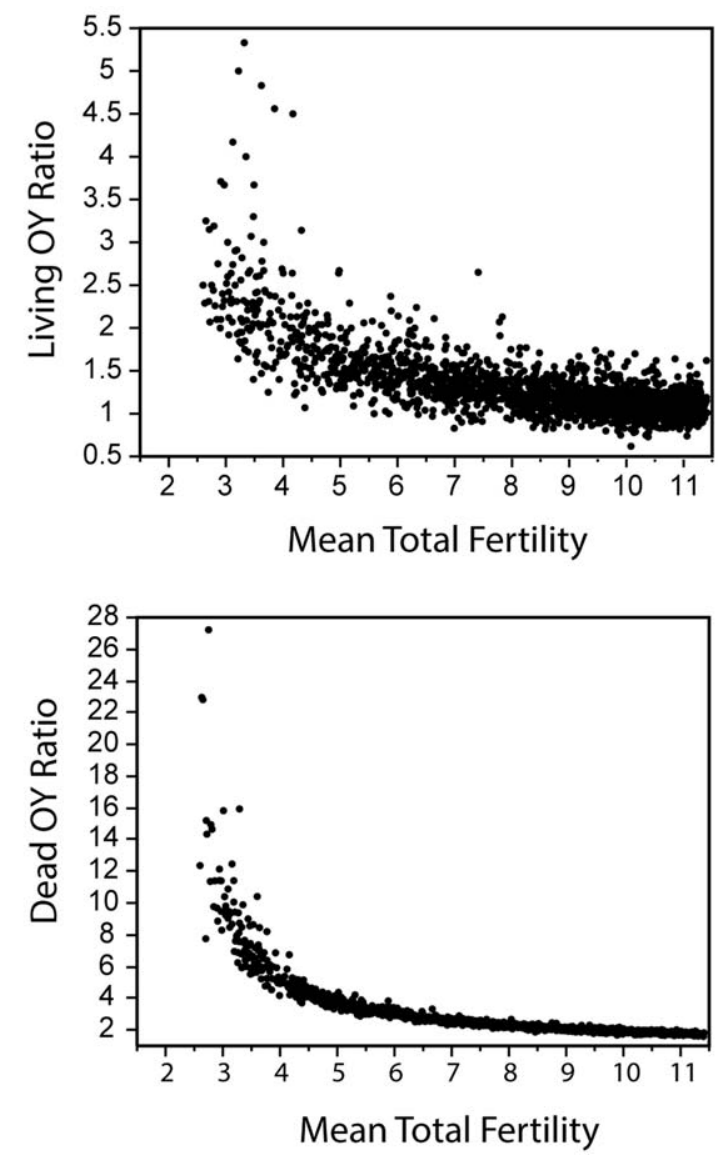

Fig. 4. Living and dead OY ratios from model populations plotted against mean total fertility and mean adult mortality experienced by those populations. 


\section{A.A. WHITE}

\section{Mortality, fertility, and the living and dead OY ratios}

Plots of the living and dead OY ratios versus the mean total fertility and adult mortality rates experienced by the living population show a set of patterned relationships varying in strength (Fig. 4). In all the four cases, relationships are generally negative: as mortality or fertility increases, the living and dead OY ratios tend to decrease.

Mortality and fertility are clearly more strongly related to the dead OY ratio than they are to the living OY ratio. These relationships are linear when both axes are displayed as logarithmic (Fig. 5). The strength of these relationships suggests that the dead OY ratio is a good predictor of mean total fertility and adult mortality across a spectrum of mortality/fertility regimes in the model populations. The living OY ratio is a weaker predictor of both adult mortality and fertility. Living OY ratios of around 1.5, for example, were associated with model populations experiencing almost the full range of mean total fertility in the model, from about 2.5 to 11 live births per female.

\section{Mean age and the living and dead OY ratios}

The mean age of the living population of each run was calculated midway through the 500-year data collection

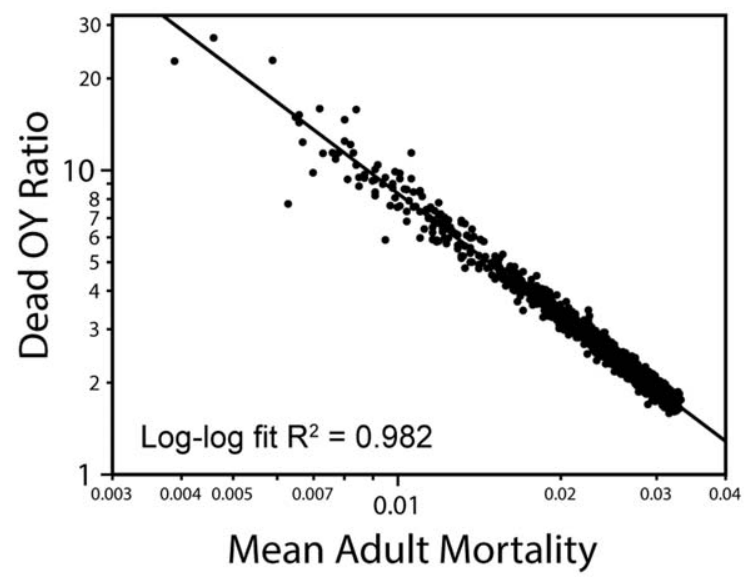

period. This provides a simple measure of longevity in the standing population. Mean age is positively related to both the living and dead OY ratios (Fig. 6), indicating that these measures are sensitive to gross patterns of age distribution that include both adults and sub-adults.

\section{Dead OY ratio and sample size}

Unlike in a model-based analysis, a dead OY ratio calculated for a skeletal or fossil assemblage will be based on a sample of individuals rather than a complete collection of all the dead individuals from the population. Sample size necessarily exerts an effect on the confidence with which we can claim we are measuring the "actual" dead OY ratio.

The assemblages of dead adult individuals produced during the course of a model run ranged in size from 188 to 21,194 . This large range was a product of variability in stable population size and adult mortality rates. At the conclusion of each run, random samples of varying size $(10,50,100,250,500,750$, and 1000) were produced from the complete list of adults who died during the 500-year data collection period. The relationships between the dead OY ratios calculated from these samples and the "actual" dead OY ratio of the complete assemblage are shown in Figure 7. Samples that exceeded the size of the complete dead assemblage were

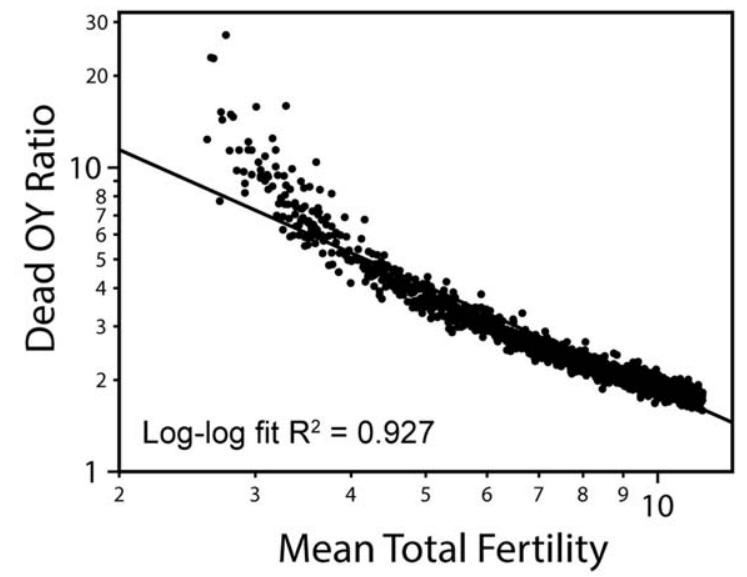

Fig. 5. Dead OY ratio plotted against mean adult mortality (left) and mean total fertility (right) on a log-log scale.
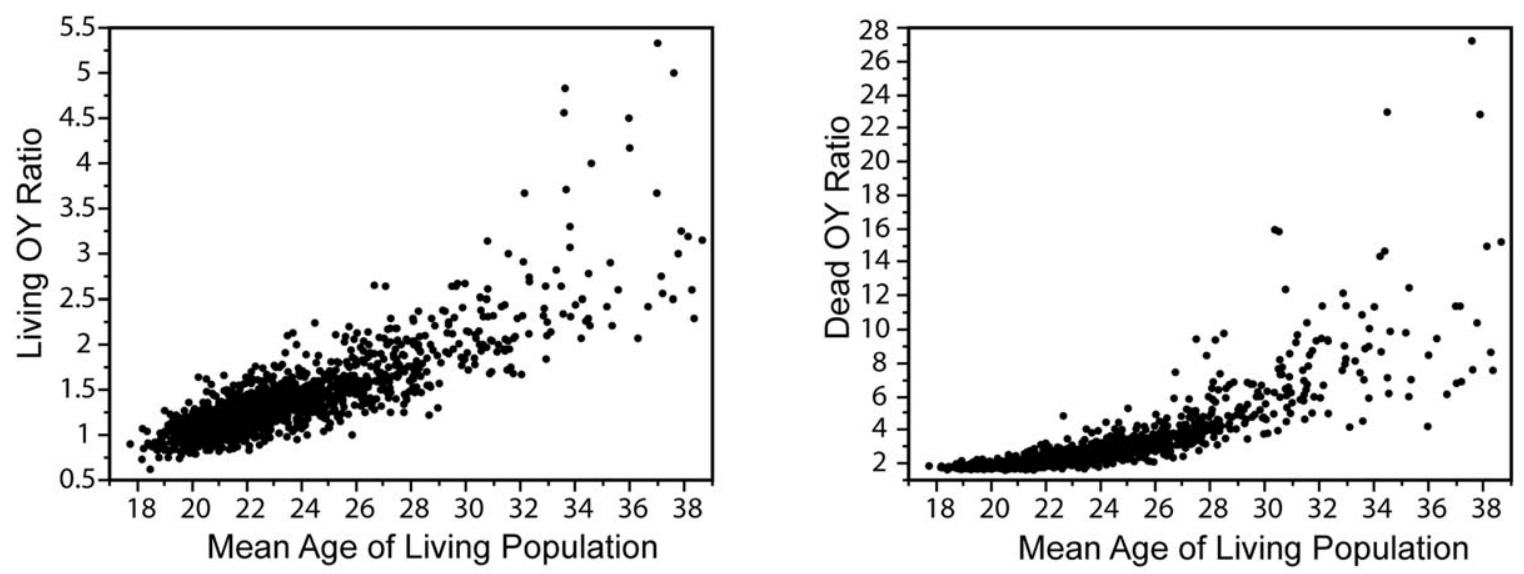

Fig. 6. Relationships between mean age and the living and dead OY ratios in model populations. 

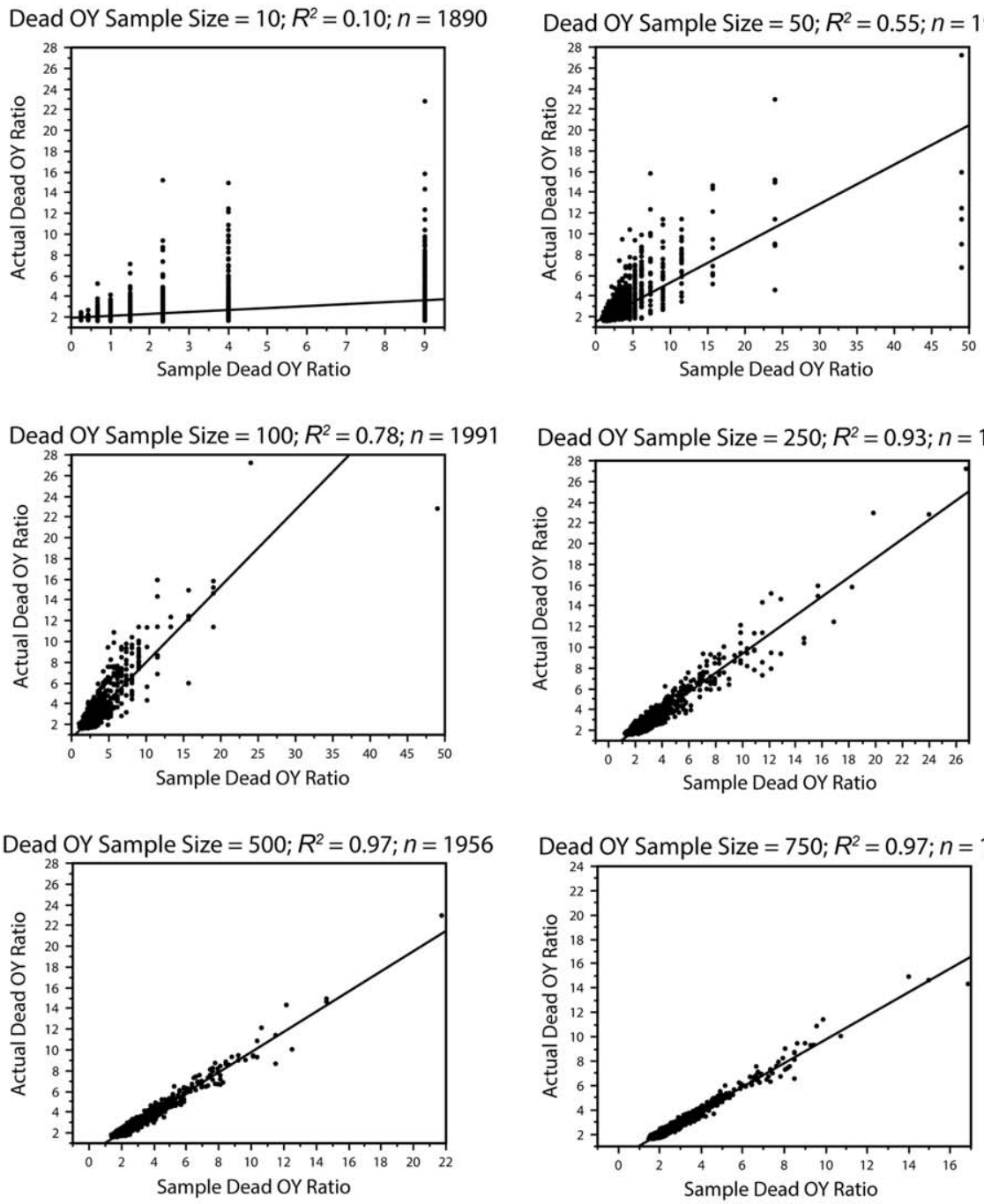

Dead OY Sample Size $=1000 ; R^{2}=0.98 ; n=1866$
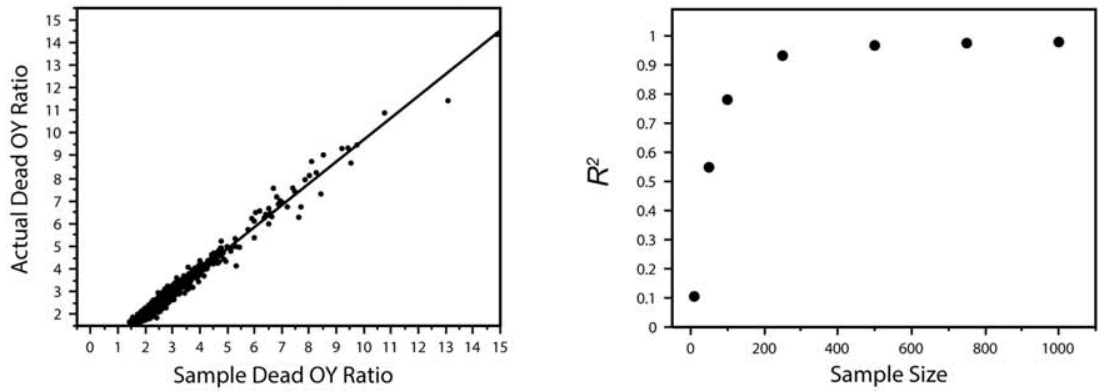

Fig. 7. Relationships between the actual dead OY ratio of a complete assemblage of dead persons from the population and the dead OY ratio calculated from samples $(n=10,50,100,250,500,750$, and 1000) of that assemblage; relationship between sample size and accuracy of estimating the dead OY ratio (characterized by value of $R^{2}$ ).

excluded. Some samples of 10 individuals were also excluded because the sample included either all old or all young adults, making calculation of a ratio impossible.

The effects of sample size on the accuracy of the dead OY ratio are straightforward (Fig. 7, bottom right). Correspondence between the sample and actual OY ratios increases rapidly as sample size grows from 10 to 250 . Much more modest increases in accuracy are obtained by doubling, tripling, or quadrupling the sample size above 250 .

\section{DISCUSSION}

Experimental results show that the dead OY ratio is related to the mean age, mean adult mortality, and mean total fertility experienced by living populations in the model. These relationships are in the expected 
directions, with higher dead OY ratios produced by living populations with a higher mean age, lower mean adult mortality, and lower fertility. The feedback mechanisms in the model limit the combinations of mortality and fertility that are possible: fertility and mortality are automatically "balanced" to maintain a stable population size. This results in a positive relationship between mean adult mortality and mean total fertility (Fig. 8), negative relationships between those variables and the dead OY ratio (see Fig. 4), and a negative relationship between the dead OY ratio and mean age (see Fig. 6).

The main issue that Caspari and Lee (2004) addressed with the OY ratio was the timing of increases in human longevity. The dead OY ratio of the early Upper Paleolithic sample (2.08) reported by Caspari and Lee (2004:10896) was higher than the dead OY ratios associated with australopithecine (0.12), early Homo (0.25), and Neanderthal (0.39) samples (Caspari and Lee, 2004:10896). This result was interpreted as reflecting a temporal trend toward increasing survivorship of older adults during the course of human evolution, with the most dramatic increase in longevity in the Upper Paleolithic (Caspari and Lee, 2004:10898). Model-based analysis clearly supports the idea that lower dead OY ratios are generally associated with populations with lower mean ages (i.e., fewer older adults), consistent with Caspari and Lee's (2004) conclusions.

It is important to note, however, that the OY ratios of the pre-Upper Paleolithic samples of Caspari and Lee (2004) were significantly lower than those associated with any of the model populations (the minimum my model populations produced was 1.59 under a wide variety of fertility and mortality conditions). The FN3D_V2 model specifically represents human behavior and physiology associated with ethnographically known huntergatherers. Several potentially interrelated factors affecting the relationships between mortality, fertility, and population age distribution (reproductive rates, maturation rates, length of the female reproductive span, cultural mating/marriage behaviors, infant/child mortality, etc.) could plausibly contribute to producing dead OY ratios less than 1.0. Although the general trends in my model data suggest that the living populations with dead OY ratios lower than 1.0 may have experienced rel-

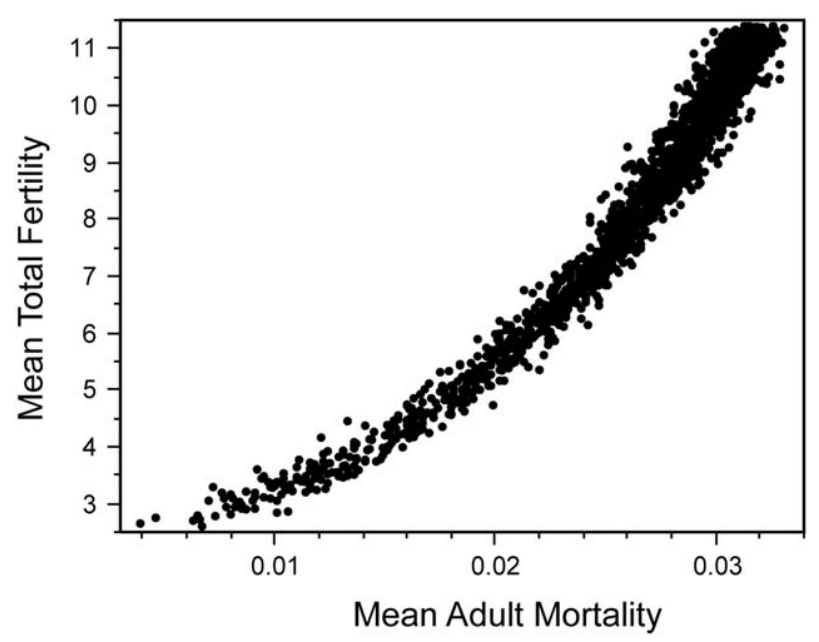

Fig. 8. Relationship between mean adult mortality and mean total fertility experienced by model populations. atively high adult mortality (presumably balanced by relatively high fertility) and been characterized by a lower mean age than the model populations, further modeling work will be required to understand how deviations from ethnographically understood "modern" conditions affect metrics such as the dead OY ratio.

Dead OY ratios around 2 (i.e., comparable to the Upper Paleolithic sample) were associated with model populations with moderate to high fertility, moderate to high mortality, and a mean age of less than 28. Given the generic, nonspatial nature of the FN3D_V2 model, it is important to use abundant caution when making inferences about the specifics of mortality, fertility, and population age structure during the Upper Paleolithic based on these results. It is also important to note that the relatively small $(n=74)$ Upper Paleolithic sample may not accurately indicate the dead OY ratio of the larger population (see Fig. 7). If the FN3D_V2 model does reasonably represent hunter-gatherer systems with demographic characteristics comparable to those of the Upper Paleolithic, it is likely that the actual Upper Paleolithic OY ratio would fall between about 1.5 and 3.5 (i.e., still significantly higher than that of the pre-Upper Paleolithic samples).

\section{CONCLUSION}

I have used an ABM to explore linkages between the dead OY ratio and the mortality, fertility, and mean age of living populations. My results suggest that differences in the dead OY ratio of skeletal/fossil populations may be a good indicator of differences in the mean age and the mortality and fertility regimes of the living populations assuming three things are true: (1) that the core mechanisms affecting mortality and fertility in the target systems were comparable to those in the model; (2) that those mechanisms are reasonably represented in the model; and (3) that samples of sufficient size are available. The ability to accurately interpret differences in the dead OY ratio in terms of changes in prehistoric mortality and/or age distribution would be contingent upon having some understanding of the conditions underlying the change. Further modeling work could be used to understand how the dead OY ratio would be expected to change under different circumstances, such as when an imbalance between mortality and fertility allows for population size to grow. Additional work will also be required to understand what changes to mechanisms and parameters of the model are required to produce low dead OY ratios like those of the pre-Upper Paleolithic samples reported by Caspari and Lee (2004, 2006).

ABMs offer a promising alternative to the "standard" equation-based approach to paleodemography. ABMs like the one used here, although "complicated" in that they have many interrelated parts, are built on rules and representations that are conceptually simple. The richness of behavior in these models emerges from the interactions of the agents in the model rather than from high-level analytical or statistical gymnastics: the alert reader will have noticed the absence of any mathematics more complicated than multiplication and division in this article. The mechanisms in these models are transparent and can be based on ethnographic data but parameterized to explore a range of conditions that is not observable ethnographically. This allows us to use 
ethnographic cases not as analogies but as construction materials for systematic theory building.

\section{ACKNOWLEDGMENTS}

The majority of the model used in this analysis was constructed during my doctoral work at the University of Michigan. Bob Whallon, John Speth, Henry Wright, and Rick Riolo served as both dissertation committee members and sources of clarity and perspective during that effort. The Center for the Study of Complex Systems at the University of Michigan supplied computational resources. Julie Lesnik provided helpful comments on an early draft of the article. I also appreciate the comments of two anonymous reviewers. I am responsible for any and all errors.

\section{LITERATURE CITED}

Angel JL. 1969. The bases of paleodemography. Am J Phys Anthropol 30:427-438.

Betzig LB. 1986. Despotism and differential reproductive success: a Darwinian view of human history. Chicago: Aldine.

Binford LR. 2001. Constructing frames of reference: an analytical method for archaeological theory building using huntergatherer and environmental data sets. Berkeley: University of California Press.

Boccara N. 2004. Modeling complex systems. New York: Springer-Verlag.

Caspari R, Lee S-H. 2004. Older age becomes common late in human evolution. Proc Natl Acad Sci U S A 101:1089510900.

Caspari R, Lee S-H. 2005a. Taxonomy and longevity: a reply to Minichillo (2005). J Hum Evol 49:646-649.

Caspari R, Lee S-H. 2005b. Are OY ratios invariant? a reply to Hawkes and O'Connell. J Hum Evol 49:654-659.

Caspari R, Lee S-H. 2006. Is human longevity a consequence of cultural change or modern biology? Am J Phys Anthropol 129:512-517.

Coale AJ, Demeny P, Vaughan B. 1983. Regional model life tables and stable populations. New York: Academic Press.

Epstein JM. 2006. Generative social science: studies in agentbased computational modeling. Princeton: Princeton University Press.

Gilbert N. 2008. Agent-based models. Quantitative Applications in the Social Sciences 153. Thousand Oaks, California: Sage Publications.

Gurven M, Kaplan H. 2007. Longevity among hunter-gatherers: a cross-cultural examination. Popul Dev Rev 33:321-356.

Hawkes K, O'Connell JF. 2005. How old is human longevity? J Hum Evol 49:650-653.
Hewlett BS. 1991. Demography and childcare in preindustrial societies. J Anthropol Res 47:1-37.

Hill K, Hurtado AM. 1996. Ache life history: the ecology and demography of a foraging people. New York: Aldine de Gruyter.

Howell N. 1979. Demography of the Dobe !Kung. New York: Academic Press.

Horowitz S, Armelagos G, Wachter K. 1988. On generating birth rates from skeletal populations. Am J Phys Anthropol 76:189-196.

Keen I. 2006. Constraints on the development of enduring inequalities in late Holocene Australia. Curr Anthropol 47:738.

Kelly RL. 1995. The foraging spectrum: diversity in huntergatherer lifeways. Washington, D.C.: Smithsonian Institution Press.

Konigsberg LW, Frankenberg SR. 1994. Paleodemography: "not quite dead." Evol Anthropol 3:92-105.

Miller JH, Page SE. 2007. Complex adaptive systems: an introduction to computational models of social life. Princeton: Princeton University Press.

Minichillo T. 2005. Paleodemography, grandmothering, and modern human evolution: a comment on Caspari and Lee (2004). J Hum Evol 49:643-645.

North MJ, Collier NT, Vos JR. 2006. Experiences creating three implementations of the Repast agent modeling toolkit. ACM Trans Model Comput Simul 16(1):1-25.

O'Connell J F, Hawkes K, Blurton Jones NG. 1999. Grandmothering and the evolution of Homo erectus. J Hum Evol 36: 461-485.

Paine RR. 1989. Model life table fitting by maximum likelihood estimation: a procedure to reconstruct paleodemographic characteristics from skeletal age distributions. Am J Phys Anthropol 79:51-61.

Pennington R. 2001. Hunter-gatherer demography.In: PanterBrick C, Layton RH, Rowley-Conwy P, editors. Hunter-gatherers: an interdisciplinary perspective. Cambridge: Cambridge University Press. p 170-204.

Roksandic M, Armstrong SD. 2011. Using the life history model to set the stage(s) of growth and senescence in bioarchaeology and paleodemography. Am J Phys Anthropol 145:337-347.

Weiss KM. 1973. Demographic models for anthropology. Memoirs of the Society for American Archaeology 27.

White AA. 2012. The social networks of early hunter-gatherers in midcontinental North America. Ph.D. thesis. Ann Arbor: University of Michigan.

White AA. 2013. Subsistence economics, family size, and the emergence of social complexity in hunter-gatherer systems in eastern North America. J Anthropol Archaeol 32:133-163.

Wobst HM. 1975. The demography of finite populations and the origins of the incest taboo. In: Population studies in archaeology and biological anthropology: a symposium. Memoirs of the Society for American Archaeology 30:75-81. 